\title{
Hemangiopericitoma retroperitoneal
}

\author{
Fernando Hidalgo Méndez ${ }^{a}$
}

\begin{abstract}
${ }^{a}$ Médico especialista en Diagnóstico por la Imagen. Servicio de Diagnóstico por la Imagen. CAP El Serral (Instituto Catalán de la Salud). Sant Vicenç dels Horts. Barcelona (España)
\end{abstract}

Correspondencia: Fernando Hidalgo Méndez (SDPI). CAP El Serral. Ctra. Sant Boi, 59-61. 08620 - Sant Vicenç dels Horts. Barcelona.

Correo electrónico:

26415fhm@comb.cat

Recibido el 23 de octubre de 2012.

Aceptado para su publicación el 24 de junio de 2013.

\section{RESUMEN}

El hemangiopericitoma es un tumor infrecuente de origen vascular que deriva de los pericitos de Zimmerman. Representa aproximadamente un $1 \%$ de todos los tumores vasculares.

En el momento del diagnóstico, establecer el potencial de malignidad de este tipo de tumoración es algo habitualmente controvertido. El pronóstico se basará en el patrón histológico y en la evolución clínica, siendo la aparición de recurrencias locales o de metástasis a distancia los únicos signos definitivos de malignidad.

El caso de un paciente de 61 años con un hemangioperictoma retroperitoneal de gran tamaño nos sirve para revisar los aspectos clínicos y patológicos, el tratamiento y los factores pronósticos de esta rara neoplasia.

Palabras clave: Neoplasias de Tejido Vascular. Hemangiopericitoma. Neoplasias retroperitoneales.

\section{ABSTRACT}

\section{Retroperitoneal Hemangiopericytoma}

Hemangiopericytoma is a rare tumour of vascular origin derived from Zimmermann's pericytes. It represents approximately $1 \%$ of all vascular tumours. At the time of diagnosis, determining the malignant potential of this type of mass is usually controversial. Prognosis will be based on histological patterns and the clinical course, with the appearance of local recurrences or distant metastases being the only definitive signs of malignancy. The case of a 61 year old patient with a very large retroperitoneal hemangiopericytoma serves to review clinical and pathological aspects, treatment and prognostic factors of this rare neoplasm

Key words: Vascular tissue neoplasms. Hemangiopericytoma. Retroperitoneal neoplasms.

\section{INTRODUCCIÓN}

El hemangiopericitoma (HPC) es una neoplasia vascular infrecuente, representando sólo el $1 \%$ del total de dichas neoformaciones ${ }^{1-3}$. Puede aparecer en cualquier parte del organismo ${ }^{1-7}$ debido a que tiene su origen celular en los pericitos de Zimmerman (células de localización ubicua, que rodean tanto a arteriolas, como a capilares y vénulas, ejerciendo una acción contráctil sobre estos vasos ${ }^{2}$ ).

Característicamente es un tumor de lento crecimiento, por lo que los signos y los síntomas clínicos suelen ser insidiosos, habitualmente derivados de la compresión que ejerce la masa tumoral sobre los órganos adyacentes ${ }^{1,5,7,8}$.

La extirpación quirúrgica completa es la piedra angular del tratamiento del HPC, generalmente precedida de una embolización de la lesión dada su característica hipervascularización ${ }^{1-3,6,7}$. Después de practicada la cirugía será necesario iniciar un seguimiento a largo plazo de los pacientes por la capacidad de esta lesión de recidivar localmente o a distancia, incluso de manera muy tardía (a veces décadas) ${ }^{1,3}$. Este seguimiento estará aún más indicado en aquellos pacientes que, en el momento del diagnóstico inicial, presentasen indicadores de un mayor potencial de malignidad. Es el propósito de este artículo hacer una especial incidencia en cuáles son estos indicadores para que el trabajo común entre los diferentes especialistas 
(clínicos, radiólogos, patólogos) nos permita ofrecer a estos pacientes un correcto diagnóstico y tratamiento y un cada vez mejor pronóstico de su enfermedad.

\section{OBSERVACIONES CLÍNICAS}

Paciente varón de 61 años de edad, con antecedentes patológicos de fumador de un paquete al día, bebedor de una unidad de bebida estándar al día, una úlcera duodenal tratada farmacológicamente a los 30 años de edad, sin antecedentes de diabetes, hipertensión arterial, dislipemias ni cardioneumopatías reseñables. El paciente refería una sensación de distensión abdominal progresiva desde hacía dos meses, con dificultad para el inicio de la micción y tendencia al estreñimiento con una deposición semanal. No explicaba vómitos, dolor abdominal, fiebre, anorexia ni pérdida de peso.

En la exploración física general destacaba tan sólo la presencia de un abdomen globuloso con una sospecha clínica de masa mesohipogástrica. La analítica general no presentaba alteraciones significativas.

La ecografía abdominal (figura 1) mostraba la presencia de una gran masa que ocupaba parte del mesogastrio y del hipogastrio, con múltiples imágenes quísticas en su interior de hasta 84 milímetros, combinadas con zonas con predominancia del componente sólido. La tomografía computerizada (figura 2) confirmó la existencia de una voluminosa masa tumoral, bien definida, de probable estirpe sarcomatosa, centrada en la raíz del mesenterio, con un componente predominantemente hipodenso y con áreas hipervascularizadas en la periferia e importante hipertrofia de los vasos intratumorales,

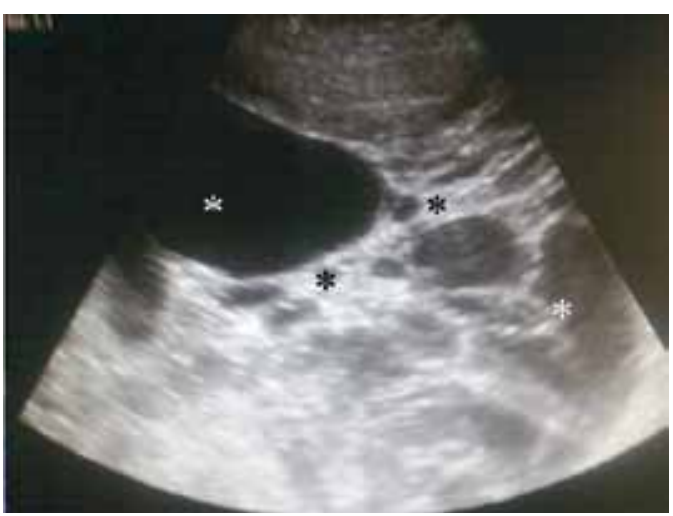

Figura 1. Ecografía abdominal. Masa que ocupa parte del mesogastrio y del hipogastrio, formada por áreas quísticas (asteriscos blancos) combinadas con zonas de predominio sólido (asteriscos negros).

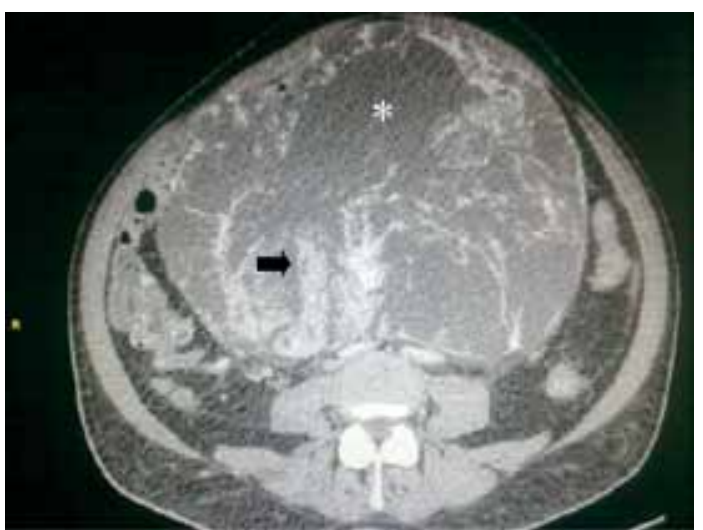

Figura 2. Tomografía computerizada abdominal. Gran masa tumoral, bien definida, con un componente predominantemente hipodenso (asterisco) y con áreas hipervascularizadas en la periferia e importante hipertrofia de los vasos intratumorales (flecha), sin signos de invasión tumoral locorregional.

con desplazamiento de estructuras en vecindad pero sin imágenes que sugirieran invasión tumoral locorregional ni signos de diseminación a distancia.

Dada la marcada vascularización tumoral, previamente a la cirugía se realizó una angiografía de la aorta abdominal y selectivas de las arterias mesentéricas superior e inferior (figura 3), en la que se procedió a la embolización de la arteria mesentérica inferior con espirales metálicas y cianoacrilato al constatarse que la vascularización principal de la masa procedía de ramas de dicha arteria.

La exéresis quirúrgica total de la lesión obtuvo una tumoración retroperitoneal de 33 centímetros

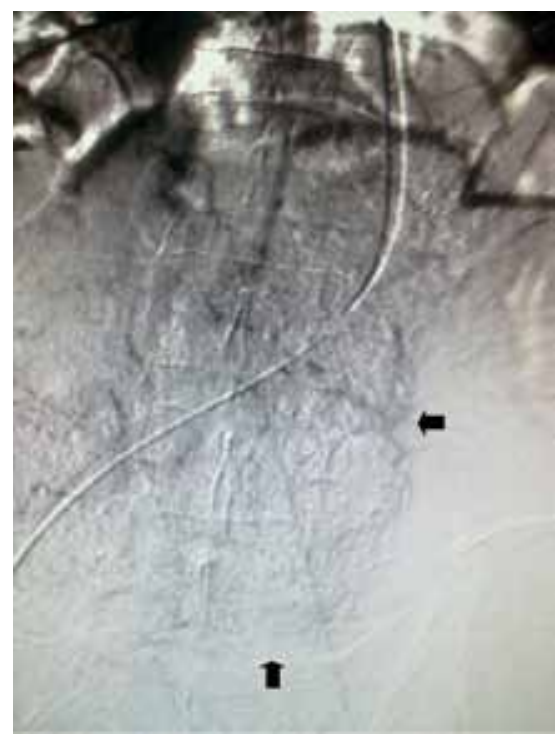

Figura 3. Arteriografía. La vascularización principal de la masa se define procedente de ramas de la arteria mesentérica inferior (flechas). 
de diámetro mayor, sólido-quística, con áreas cavitadas, con zonas necróticas y zonas hemorrágicas. El diagnóstico histológico fue de hemangiopericitoma del retroperitoneo, con rasgos morfológicos de comportamiento maligno.

\section{COMENTARIOS}

Desde que en 1942 Stout y Murray describieron por primera vez el $\mathrm{HPC}^{9}$, la distinción entre benignidad o malignidad en esta rara neoplasia vascular ha sido motivo de controversia. Hoy en día se considera la presencia de una serie de datos histológicos y clínicos como indicativos del potencial de malignidad de la lesión. Así, su conocimiento será capital a la hora de establecer el pronóstico y el protocolo de seguimiento en estos pacientes.

El HPC representa alrededor del $1 \%$ de todos los tumores vasculares ${ }^{1-3}$ y el $5 \%$ de todos los sarcomas de tejidos blandos ${ }^{1-4}$. Las dos principales localizaciones del HPC son las extremidades inferiores (especialmente el muslo) y el espacio retroperitoneal, aunque también se ha descrito en otras diversas localizaciones (entre ellas: pelvis, cabeza, cuello, tronco, extremidades superiores, meninges, útero y bazo) ${ }^{1-7}$. No tiene una mayor prevalencia en ninguno de los dos $\operatorname{sexos}^{1-3,7} \mathrm{y}$, aunque su rango de edad de presentación es muy amplio, suele ocurrir más en adultos jóvenes (con una media de 45 años) $)^{1-3,5-7}$.

Los HPC retroperitoneales (así como otros muchos tumores primarios de este espacio anatómico) suelen tener una presentación clínica insidiosa, en la que es habitual que exista una marcada disparidad entre un gran tamaño tumoral y una relativa escasez de síntomas. Ello se debe a que el retroperitoneo es un espacio al cual el HPC se va adaptando durante su lento crecimiento, pudiendo permanecer clínicamente silente durante tiempo ${ }^{8}$. La sintomatología vendrá marcada por la compresión o invasión de órganos y estructuras adyacentes, así como por la palpación de la propia masa tumoral ${ }^{1,5,7,8}$. Además, el HPC en ocasiones se asocia a algunos síndromes paraneoplásicos (ginecomastia, crisis hipoglucémicas, hipertensión y osteomalacia), los cuales revierten tras la extirpación de la lesión ${ }^{1,3,7}$. Cuando nuestro paciente acudió por primera vez a la consulta, el tamaño de la lesión era ya considerable, presentando una moderada distensión abdominal y síntomas derivados de la compresión del tracto urinario (dificultad para iniciar la micción) y del tubo digestivo (tendencia al estreñimiento).
El diagnóstico de HPC sólo se obtiene de manera concluyente mediante el estudio histológico ${ }^{1,2}$. Las pruebas de imagen no proporcionan ningún dato patognomónico de HPC, pero nos ofrecen una valiosa información prequirúrgica no sólo para definir el tamaño y las características morfológicas de la lesión, sino también para evaluar una potencial afectación de los órganos y estructuras adyacentes ${ }^{1,2}$. La tomografía computerizada generalmente muestra el HPC como una masa de gran tamaño, lobulada, bien circunscrita, con características de tejido blando, que puede contener calcificaciones y áreas hemorrágicas, quísticas o de necrosis, así como una típica hipervascularizacón en su periferia que se constata tras la administración de contraste endovenoso ${ }^{1-3,7}$. La angiografía tendrá una doble utilidad: confirmar la hipervascularización de esta neoplasia y, una vez identificados los vasos nutricios de la lesión, proceder a su embolización para así disminuir las posibles complicaciones hemorrágicas del acto quirúrgico subsiguiente ${ }^{1,2,6}$.

La biopsia percutánea de estas lesiones no se aconseja al ser de riesgo por la hipervascularización tumoral ${ }^{1,6}$.

El tratamiento de elección del HPC retroperitoneal es la resección quirúrgica completa ${ }^{1-4,6-8}$, generalmente precedida de una embolización selectiva de los principales vasos tumorales practicada durante una angiografía ${ }^{1-3,6,7}$. La radioterapia puede ser útil como terapia post-quirúrgica para disminuir el riesgo de recidiva local ${ }^{1,2,7}$, aunque su uso aislado no reporta beneficios significativos ${ }^{2}$. La quimioterapia sólo muestra una limitada utilidad en el HPC metastásico, obteniendo remisiones parciales únicamente en algunos pacientes ${ }^{2}$.

En esta comunicación se pretende hacer especial énfasis en la dificultad que supone establecer un diagnóstico de benignidad o de malignidad cuando nos encontramos ante un HPC. Esta distinción se orientará en base a unos criterios clínicos y a unos criterios histológicos, indicándonos ambos acerca del pronóstico y del seguimiento de cada paciente ${ }^{1-3,6}$.

Desde el punto de vista de los datos clínicos, la invasión de estructuras adyacentes al tumor en el momento del diagnóstico inicial indica un comportamiento agresivo ${ }^{1}$, siendo los únicos signos definitivos de malignidad la presencia de recurrencias locales y la aparición de metástasis a distancia, en ambos casos pudiendo aparecer incluso décadas más tarde ${ }^{3}$. El tiempo medio hasta la aparición de recurrencias locales se 
ha establecido en 17 meses $^{1}$, precediendo a la posterior detección de metástasis a distancia en las dos terceras partes de estos pacientes con recidiva local ${ }^{1,6}$. Las metástasis ocurren por vía hematógena o por vía linfática, afectando principalmente al pulmón, hígado y huesos ${ }^{1,6}$. El tiempo que pueden tardar en aparecer las metástasis a distancia es muy variable, con un media entre 4 y 5 años después del diagnóstico inicial de la neoplasia ${ }^{1}$.

Por su parte, el estudio histológico sugiere un mayor potencial de malignidad cuando se observa una importante actividad mitótica, una elevada celularidad, focos de hemorragia o de necrosis o la presencia de anaplasia celular ${ }^{1-3,6}$.

El pronóstico de esta neoplasia mejora de manera clara cuando el paciente es sometido a una cirugía radical. En el HPC se describen tasas globales de supervivencia a los 5 años de un $71 \%$ y a los 10 años de un $54 \%$, pero variando significativamente la tasa a los 5 años entre el $79 \%$ cuando la exéresis de la lesión fue completa y el $50 \%$ cuando no lo fue ${ }^{4}$.

En resumen, el HPC es una rara neoplasia de origen vascular en la que, una vez realizado el tratamiento quirúrgico radical como terapia de elección, pueden aparecer recidivas locales o metástasis a distancia años o incluso décadas más tarde del diagnóstico inicial. El conocimiento de los criterios clínicos y patológicos descritos en este artículo como sugestivos de un potencial de malignidad nos debe servir a clínicos y a radiólogos para proporcionar a estos pacientes un mejor pronóstico de supervivencia en base a un correcto seguimiento a muy largo plazo de su enfermedad.

\section{BIBLIOGRAFÍA}

1. Lema J, Cimadevila A, Rodríguez H, Durana C, Blanco M. Hemangiopericitoma retroperitoneal gigante. Actas Urol Esp. 2001; 25 (9): 672-5.

2. Chan SC, Lee CM, Ng YB, Tsai CH. Giant retroperitoneal hemangiopericytoma. Postgrad Med J. 1995; 71: 692-700.

3. Yin B, Liu L, Li Y, Geng D, Du Z. Retroperitoneal hemangiopericytoma: case report and literature review. Chin Med J. 2011; 124 (1): 155-6.

4. Bajracharya A, Gupta R, Agrawal CS, Karki S. Hemangiopericytoma in the retroperitoneal space. Health Renaiss. 2011; 9 (3): 210-3.

5. Espat NJ, Lewis JJ, Leung D, Woodruff JM, Antonescu CR, Shia $\mathrm{J}$ et al. Conventional hemangiopericytoma: clinical outcome. Cancer. 2002; 95 (8): 1746-51.

6. Slupski M, Piotrowiak I, Wlodarczyk Z. Local recurrence and distant metastasis 18 years alter resection of the greater omentum hemangiopericytoma. World J Surg Oncol. 2007; 5: 63.

7. Martín I, Vidal R, Blanco R, Martín A. Caso radiológico para diagnóstico: hemangiopericitoma pélvico. Rev Chil Radiol. 2011; 153: 195-7.

8. Virseda JA, Donate MJ, Pastor H, Carrión P, Martínez J, Martínez $C$ et al. Tumores retroperitoneales primarios: revisión de nuestros casos de los diez últimos años. Arch Esp Urol. 2010; 63 (1): 13-22.

9. Stout AP, Murray MR. Hemangiopericytoma: vascular tumor featuring Zimmerman's pericytes. Ann Surg. 1942; 116: 26-35. 